TABLE 3.

\begin{tabular}{|c|c|c|c|}
\hline$p \mathbf{H}$ & $1 \cdot 0$ & $5 \cdot 0$ & $10 \cdot 5$ \\
\hline $\begin{array}{l}\% \text { Change in work: } \\
\text { Formaldehyde treated } \\
\text { Buffer treated }\end{array}$ & $\begin{array}{r}+11 \cdot 0 \\
+1 \cdot 0\end{array}$ & $\begin{array}{l}+6 \cdot 0 \\
-1 \cdot 0\end{array}$ & $\begin{array}{r}-58 \cdot 0 \\
-61 \cdot 0\end{array}$ \\
\hline
\end{tabular}

On comparing these results with those in Table 2, it will be seen that no simple relationship exists between the effect of new linkage formation on the resistance of fibres to extension and on the supercontraction of fibres in boiling solutions of sodium metabisulphite.

University,

Leeds.

July 12.

1 Stoves, J. L., Trans. Faraday Soc., 39, 294 (1943).

"Britton, H. T. S., "Hydrogen Ions", 217-223 (London, 1932).

${ }^{8}$ Stoves, J. L., Trans. Faraday Soc., 38, 254 (1942).

'Speakman, J. B., J. Soc. Dyers and Col., 50, 341 (1934).

\section{The Concept of Force}

Mr. JoshuA C. Gregory's statement" "When a motor-car turns sharply round a corner the passenger feels as if he were shoved but not as if a thing shoved him" suggests that he has not clearly analysed the experience. The main sensations (other than visual) are of contact and pressure on the side of the body on the outside of the curve; in other words, of a force as ordinarily experienced acting towards the centre, in the same direction as the acceleration. The sensations are exactly similar to those felt in the back when a car accelerates rapidly. If there is no contact there is no sensation and no force, and the passenger continues in his previous uniform motion in accordance with Newton's First Law. In the same way, with high acceleration the head and shoulders which are not in contact with the back of the seat move relatively backwards over the top of this and experience no sensation of force.

Manchester Grammar School, Manchester, 13.

'Nature, 154, 24 (1944).

W. B. YAPP.

Mr. Joshua C. Gregory's letter under this title ${ }^{1}$ seens to me misleading. A "notion of force" without a "notion of matter" appears to me a contradiction. Without matter there can be no motion and therefore no acceleration; and without acceleration there can be no force since force denotes a massacceleration product.

When I go round a sharp corner in a motor-car I definitely experience the sensation of being shoved by the seat.

To describe forces as "immortal" or "imperceptible" strikes me as idealist nonsense. A force lasts just as long as the matter with which it is associated has acceleration; the force associated with the retardation of a hammer on one's thumb is perceptible enough. "Disembodied force" is as meaningless as "matterless motion". Let us keep these anthropomorphic and idealist notions of force out of physics ; force would exist in a lifeless universe.

Clearbrook,

JoHn CASE.

Nr. Yelverton,

S. Devon.

${ }^{1}$ Nature, 154, 24 (1944).
THE description of force, gathered from the "Hermetica" into my communication in Nature of July 1, p. 24, does not, as such, ask Mr. John Case to believe in "immortal" and "imperceptible" forces, or to adopt any "anthropomorphic and idealist notions". In 1864 Colding connected immaterial and imperishable forces with the perpetuity of energy which had been clear to him since 1840, and there is at least a hint at the modern conservation of energy in the immortal forces of the "Hermetica". Those who still refuse to reduce causal efficacies to mere regulat routine can sympathize with the working of the Hermetical forces. The letter, however, only suggests that the Hermetical concept of force embodies a recognizable distinction in sensory experience. The concept can do this however violently modern thought disclaims it in other respects.

Muscular sensations themselves, the suggestion runs, only produce a sense of what Prof. Price calls "pure force, disembodied force as it were". This corresponds to the incorporeal nature of the Hermetical force. Sensations of contact are essential to complete the sense of the resistant or propulsive material thing. The Hermetical forces only work in bodies; this corresponds to the very usual combination of muscular and contact sensations. Cut out a man's contact sensations, leave all his other sensations in, he will then, according to the suggestion, have only a sense of "disembodied force" and have no sense of material things. If this sense of pure or disembodied force is actually felt in the centrifugal experience, as Price suggests, the phrase "disembodied force" is not "meaningless", as Mr. Case asserts.

Mr. W. B. Yapp concentrates on a denial of this sense of pure force when a motor-car corners rapidly or accelerates sharply. When a motor-car turns quickly, a passenger on the back seat is thrown somewhat laterally and feels, or may feel, as if an immaterial force throws him, as if he is shoved without being shoved by a material shover. This is the experience of the writer and presumably of Prof. Price. Some people stress unexpectedness-they have this sense of pure force when the car takes them unawares as it corners quickly. They have a similar experience during a sharp acceleration backwards or forwards. There seem also to be analogous aeroplane experiences. When I am 'centrifuged" in a motor-car I do not "definitely experience the sensation of being shoved by the seat", as Mr. Case does, nor do I sense a contact push on my free side when I am pressed against the resistant side of the car, as Mr. Yapp seems to do.

Some people at least confess to a momentary feeling of "what on earth is happening!" and an evanescent whiff of uncanniness sometimes rushes through me. Mr. Case and Mr. Yapp may dissent because the inveterate tendency to connect pushes or pulls with contacts predisposes them against a recognition of the actual, though usually momentary, experience. Many 'centrifuged, passengers do seem to glimpse, if only for a moment, the sense of disembodied forces that might be habitual if there were no contact sensations. The experience seems to occur, however the somewhat elusive concept of force is finally assessed.

Prof. Price, of course, though he gave me a cue, is not responsible for the use made of it.

Mount Hotel, Clarendon Road. Joshua C. Gregory, Leeds, 2. 